\title{
A population statistics approach for safety assessment in robotic cochlear implantation
}

\section{Running Title: Safety in Robotic Cochlear Implantation}

Dr Tom Williamson $\mathrm{PhD}^{1}$, Dr Kate Gavaghan $\mathrm{PhD}^{1^{*}}$, Dr Nicolas Gerber $\mathrm{PhD}^{1}$, Dr Stefan Weder $\mathrm{MD}^{2}$, $\mathrm{Dr}$ Lukas Anschuetz² MD, Dr Franca Wagner MD³, Dr Christian Weisstanner MD³, Dr Georgios Mantokoudis $M D^{2}$, Prof. Marco Caversaccio MD², Prof. Dr-Ing Stefan Weber $\mathrm{PhD}^{1}$

1) ARTORG Center for Biomedical Engineering Research, University of Bern, Bern, Switzerland

2) Department of Otorhinolaryngology, Head and Neck Surgery, Inselspital, Bern University Hospital, Bern, Switzerland

3) Institute for Diagnostic and Interventional Neuroradiology, Inselspital, Bern University Hospital, Bern, Switzerland

Funding: This work was completed as part of the Nano-Tera initiative; the work was scientifically evaluated by the Swiss National Science Foundation, financed by the Swiss Confederation and funded by NanoTera.ch

* Corresponding Author: Kate Gavaghan, ARTORG Center for Biomedical Engineering Research, University of Bern, Murtenstrasse 50, 3008, Bern, Switzerland, kate.gavaghan@artorg.unibe.ch, Tel: +41 31632 3754, Fax: +4131632 7576 


\section{Abstract}

\section{Hypothesis}

3 Descriptive statistics with respect to patient anatomy and image guidance accuracy can be utilized to 4 assess the effectiveness of any system for minimally invasive cochlear implantation, on both an $5 \quad$ individual patient and wider population level.

\section{$6 \quad$ Background}

7 Minimally invasive cochlear implantation involves the drilling of a tunnel from the surface of the mastoid 8 to cochlea, with the trajectory passing through the facial recess. The facial recess anatomy constrains 9 the drilling path and places prohibitive accuracy requirements on the utilized system. Existing single 10 thresholds are insufficient for assessing the effectiveness of these systems.

\section{Methods}

A statistical model of the anatomical situation encountered during minimally invasive drilling of the mastoid for cochlear implantation was developed. A literature review was performed to determine the statistical distribution of facial recess width; these values were confirmed through facial recess measurements on CT data. Based on the accuracy of a robotic system developed by the authors, the effect of variation of system accuracy, precision and tunnel diameter examined with respect to the potential treatable portion of the population.

\section{Results}

A facial recess diameter of $2.54 \pm 0.51 \mathrm{~mm}(\mathrm{n}=74)$ was determined from a review of existing literature; subsequent measurements on CT data revealed a facial recess diameter of $2.54 \pm 0.5 \mathrm{~mm}(n=23)$. The developed model demonstrated the effects of varying accuracy on the treatable portion of the population.

\section{Conclusions}

The presented model allows the assessment of the applicability of a system on a wider population scale beyond examining only the system's ability to reach an arbitrary threshold accuracy. 


\section{Introduction}

The enabling of minimally invasive cochlear implantation ( $\mathrm{MICl}$ ) utilizing robotic and image guidance technology has been the focus of significant research efforts over the last decade. The approach involves drilling a tunnel directly from the surface of the mastoid to the cochlea, removing the need for the traditional mastoidectomy, in which a portion of the mastoid is milled away to allow the visualization of structures for the safe creation of a posterior tympanotomy and access to the cochlea for electrode insertion. Previously investigated approaches to the procedure include hand held guidance using standard surgical navigation techniques ${ }^{1}$, patient specific templates ${ }^{2}$, and image-guided robotics utilizing modified industrial ${ }^{3}$ and custom manipulators in serial ${ }^{4}$ or parallele ${ }^{5,6}$ configurations.

The major technical challenge of the procedure is accuracy, with newly developed technologies routinely reporting achieved drilling accuracy results ${ }^{1-6}$. However, it remains unclear how these values relate to clinical practice, with existing accuracy reports often referring to a previously defined requirement of $0.5 \mathrm{~mm}$ or better at a target on the cochlea ${ }^{1}$. This threshold does not sufficiently reflect the complexity related to safety aspects that are applicable both on a population and individual patient level. Due to significant variations in patient anatomy, general claims of applicability and safety based on a "one-size-fits-all" threshold are insufficient with respect to an image-guided system for MICl.

The decision about whether the system can be applied in a particular case should be made based on the specific anatomy of the patient and the known accuracy of the system, investigated in clinically relevant and sufficiently statistically powered experiments. Furthermore, it would be of interest to investigate the applicability of a system on a population level beyond its ability to reach an arbitrary threshold. For these reasons, we propose to build upon the concept introduced by $\mathrm{Nau}^{7}$ in which the ability of a robot to perform a surgical task within required tolerances was assessed based on industrial standards for manufacturing. Applied to $\mathrm{MICl}$, required tolerances can be specified based on patient anatomical information.

Subsequently, a method that, based on a given system's descriptive accuracy statistics, can compute the treatable portion of the population using available statistical information on the facial recess and estimate the likelihood of a safe procedure given an individual patients anatomy and system configuration, was developed and is presented herein. The developed model was then utilized to evaluate the theoretically treatable portion of the population utilizing our own investigational robotic 
system ${ }^{4}$, besides a number of arbitrary alternative systems to demonstrate how the technique can be utilized to analyze the applicability of any future developed system.

\section{Materials \& Methods}

\section{A Statistical Model for Patient Treatability Assessment}

The desired model requires information about both the size of the facial recess and the specific system utilized. First, one can define a safe region based on the specific characteristics of the system in use. If a drill bit with a known diameter is utilized as part of a system with a characterized level of accuracy, such a safe region can be defined as a circle as shown in Figure 1, with a radius defined as in (1).

$$
r_{\text {safe }}=\frac{\emptyset_{\text {Drill }}}{2}+\mu_{\text {acc }}+n \cdot \sigma_{\text {acc }}
$$

whereby $r_{\text {safe }}$ is the radius of the safety region, $\emptyset_{\text {Drill }}$ is the drill diameter, $\mu_{\text {Acc }}$ the system mean accuracy, $\sigma_{A c c}$ the standard deviation of the system accuracy and $n$ defines the rate at which the drilled trajectory will fall within this safety region (i.e. the number of standard deviations).

If one then considers the facial recess diameter of a specific patient as well as the specific distribution of the system accuracy, the percentage level at which a specific facial recess diameter intercepts with the safety region can be calculated. Thus, for a specific patient a "confidence level" can be defined based on the cumulative distribution function (CDF). Note that as a safety region cannot be defined for a facial recess diameter of less than that of the drill diameter $\left(F R_{e f f} \leq 0\right)$, the normal CDF must be altered to describe the folded CDF as in (2), for $F R_{e f f} \in[0, \infty)$.

$$
F\left(F R_{e f f} \mid \mu_{a c c}, \sigma_{a c c}\right)=\frac{1}{2}\left[\operatorname{erf}\left(\frac{F R_{e f f}+\mu_{a c c}}{\sigma_{a c c} \sqrt{2 \pi}}\right)+\operatorname{erf}\left(\frac{F R_{e f f}-\mu_{a c c}}{\sigma_{a c c} \sqrt{2 \pi}}\right)\right]
$$

Whereby $F(x \mid \mu, \sigma)$ is the folded normal CDF, $\mu_{a c c}$ and $\sigma_{a c c}$ are the mean and standard deviation of the system accuracy, $F R_{\text {eff }}$ is the effective radius of the facial recess, described as the measured radius of the facial recess minus the radius of the drill, and erf is the Gauss error function. Thus, this function will provide the probability that the drill will fall within a circle with a diameter of $F R_{\text {eff }}$.

The model described mathematically above and graphically in Figure 1 allows the definition of a confidence level for a specific patient, and can be extended to estimate the minimum facial recess 
diameter which a system can treat with a certain confidence level. In order to allow this information regarding two factors is required: the facial recess diameter and the specific accuracy statistics of the system to be evaluated. The definition of these parameters is described in the following sections.

\section{Determination of Facial Recess Diameter}

The facial recess is bounded posteriorly by the facial nerve, anteriorly by the posterior annulus of the tympanic membrane or chorda tympani and superiorly by the ossicles (Figure 2). The dimensions of the facial recess (and extended facial recess excluding the chorda tympani) have been described in a number of previous publications, utilizing a variety of techniques including measurements on medical image (CT) data ${ }^{8,9,10}$, histological specimens ${ }^{11,12,13}$, and physical measurements on extracted bone samples ${ }^{14,15,16}$. All previously described dimensions of both the facial recess and extended facial recess are reported in Table 1.

With respect to our target scenario, we consider measurements of the facial recess at the level of the round window or basal turn of the cochlea to be most relevant. Only these values were included in this study (see Table 2). Subsequently, the mechanical measurements utilizing a caliper after skeletonization of the chorda tympani and facial nerve ${ }^{16}$, measurements in medical image data after $\mathrm{MICl}$ drilling ${ }^{10}$ and histological measurements ${ }^{12}$ will be utilized for further analysis. While the methodologies utilized vary, each represents measurements of the same anatomical region and thus similar values can be expected.

The dimensions of the facial recess derived from literature were subsequently confirmed in a study using a total of 23 temporal bone specimens. High resolution CT scans of the specimens were acquired (Siemens Somatom Definition Edge, $0.156 \times 0.156 \times 0.2 \mathrm{~mm}^{3}$ ) and the relevant structures segmented utilizing a custom planning software ${ }^{17}$. A trajectory was defined from the mastoid surface to the round window through the facial recess, with the trajectory position optimized to maximize the distance between the drill and the anatomy. The size of the facial recess was then calculated by summing the trajectory diameter and the distance between the surface of the trajectory tunnel to the chorda tympani and facial nerve respectively (Figure 2). 
The potentially treatable patient population can be evaluated according to (2) by assessing the facial recess diameter of the population and utilizing available descriptive accuracy statistics of a given system. Additionally, effects on the potentially treatable patient population as a result of alterations in the system (i.e. accuracy, drill diameter) can be investigated. Consequently, we define four potentially valid system configurations for further analysis as follows:

Additionally, we consider the effect of varying tunnel diameters; alternative diameters of $1.4 \mathrm{~mm}$ (lower boundary to accommodate an electrode), 1.6 and $2.0 \mathrm{~mm}$ (upper bound given by the anatomical situation) were considered. The effects of altering the tunnel diameter and system accuracy are assessed separately based on the system accuracy $\mathrm{X}_{0}$; a total of 7 systems are thus evaluated.

\section{Results}

\section{Determination of Facial Recess Diameter}

The width of the facial recess was successfully determined in all 23 samples, with a mean diameter of $2.54 \pm 0.5 \mathrm{~mm}$ derived. The history of the specific temporal bones is unknown, however previous studies have shown no significant differences between the size of the facial recess in adults and children ${ }^{18}$ and between subjects of different races ${ }^{15}$. The calculated values were found to be in close agreement with those observed in previous $\mathrm{CT}$, histologic and physical evaluation.

The combination of the most relevant measurements from literature (i.e. those of the facial recess at the level of the round window or basal turn of the cochlea) and the data extracted from this work reveals a mean facial recess diameter of $2.54 \pm 0.5 \mathrm{~mm}$, with a total sample size of 97 samples (Table 2); this value was utilized for all subsequent calculations. No statistically significant difference in facial recess diameter was observed based on the analysis method utilized (unpaired t-test, $\mathrm{p}=0.05$ ). 
The developed methods allowed evaluation of the relationship between the specified system accuracy and facial recess size for both the base system and hypothetical systems.

The statistical probability that a system with a given accuracy and drill diameter will drill within a given radius can be represented graphically as shown in Figure 3, in which the facial recess diameter of the population in the range of 2 standard deviations from the mean is shown.

Utilizing system $\mathrm{X}_{0}$, approximately $46.7 \%$ of the population can accommodate a safety region of three standard deviations above the mean drilling error, when utilizing a drill diameter of $1.8 \mathrm{~mm} ; 60 \%$ and $71 \%$ of the population can accommodate safety regions covering $2 \sigma$ and $1 \sigma$.

With respect to the defined theoretical systems, the accuracy and precision variations of the systems demonstrates the effects these values have on the size of the system safety region and subsequent effects on the treatable portion of the population (Figure 3, Table 3). With a defined threshold of three standard deviations, the effect of changes in the precision of the system tend to dominate.

\section{Discussion}

$\mathrm{MICl}$ has been the subject of significant research over the past decade, culminating in the first clinical trials in $2013^{19}$. Given the delicate nature of the procedure, particularly the lack of direct visual feedback and proximity to the facial nerve, a more complex model for the assessment of the system performance both on a population level, as well as on an individual patient level, is required. To this end, the definition of inclusion criteria for $\mathrm{MICl}$ must reflect the fact that the integrity of the facial nerve is of the highest priority. Thus, we suggest that accuracy assessments using a "one size fits all" threshold are potentially misleading. As the incidence of permanent FN injury reported in the literature for conventional $\mathrm{Cl}$ surgery is approximately $0.1 \%{ }^{20}$, a robotic approach should remain, at minimum, equally safe with respect to this value. We therefore suggest the use of a 3-sigma level, representing approximately $99.9 \%$ of cases drilled potentially within the safe region of the facial recess. While a threshold of $3 \sigma$ limits the number of potentially treatable patients (an inclusion rate of $47 \%$ is expected for initial clinical trials using system $\mathrm{X}_{0}$ ) such a threshold should serve to increase procedural safety. of cases, it is important to note that this value cannot be inverted to assess the possible rate of nerve 
damage because the higher error in the remaining cases will not always be directed towards one of the bounding nerves (facial nerve or chorda tympani). The definition of a safe region based on the characterized accuracy does therefore not provide any information about how likely the system is to actually damage any specific structure, only the probability that the drill will fall within a safe region.

Furthermore, the statistical model described above assigns equal weighting to both the chorda tympani and facial nerve, while in reality the surgeon is much more likely to sacrifice the chorda tympani (up to $20 \%$ of cases $^{21}$ ) during traditional cochlear implant surgery. This may be reflected during planning of the trajectory, which rarely passes through the center of the facial recess, but is instead offset away from the facial nerve. Extension of the model to account for these factors is relatively simple: if one calculates the distance corresponding to the specific change in desired probability, this can then be added on to the effective facial recess diameter. As an example, if a confidence level of one standard deviation is acceptable at the chorda tympani, two standard deviations can be added to the effective facial recess diameter for the calculation. In reality, the three sigma safety region remains, but with the center of the trajectory shifted such that the region overlaps with the border of the chorda tympani at a level of one standard deviation. In the baseline case, this would increase the treatable portion of the population to approximately $60 \%$. Additionally, although a diameter of $1.4 \mathrm{~mm}$ is currently required such that an electrode can be inserted through the tunnel, future developments may include reductions in electrode diameter and thus allow smaller tunnels, leading to increases in the treatable portion of the population.

The facial recess measurements obtained adhere closely to data derived in previous studies; consequently we conclude that the available data on the width of the facial recess is valid and that a value of $2.54 \pm 0.5 \mathrm{~mm}$ can be accepted as representative. The current approach assumes that patient anatomy is normally distributed, while the CT data utilized for analysis suggests that anatomy may be skewed slightly positively (i.e. towards larger diameters), a normal distribution will provide a good, if slightly low, initial estimation of patient treatability. If future evaluation of the anatomy reveals significant variation from the assumed normal model, the approach can be relatively easily modified to reflect the new distribution. With respect to the identification of anatomical structures, errors in segmentation may lead to under- or overestimation of facial recess diameter and any image-guided system relies on the accuracy of the underlying image-based plan. Subsequently, precise and reproducible identification of anatomical structures within medical image data is imperative to ensuring the integrity of the anatomy. 
Furthermore, the approach assumes that the facial recess is the major limiting factor with respect to trajectory placement. In our experience this has been the case, however there may exist patients where trajectory placement is limited by other anatomical features (for example the dura or sigmoid sinus). Other limiting factors may include the alignment of the trajectory with the scala tympani, however one of the advantages of the approach is that it allows insertion angles to be optimized pre-operatively, thus reducing the likelihood of insertion failure due to large angles. While these factors could subsequently decrease the number of treatable patients, we expect that the numbers would not significantly impact the statistical results described above.

Finally, one must consider that regardless of the described system accuracy the possibility still remains for cases in which, due to failures of the system or unexpected errors, the drilling accuracy falls well outside the expected distribution. Subsequently, a number of additional safety approaches have been investigated, including methods based on observed drilling forces ${ }^{22}$, endoscopic imaging ${ }^{23}$ and intraoperative $\mathrm{CT}^{24}$. Additional protection systems such as facial nerve monitoring ${ }^{25}$ and heat modeling ${ }^{26}$ have also been explored. Each of these methods increase the safety of the procedure by providing measurement redundancy using independent physical effects and thus their utilization should be strongly encouraged whenever considering clinical application.

\section{Conclusions}

This work has presented the development and evaluation of a statistical model for the assessment of $\mathrm{MICl}$ systems' safety and effectiveness. A facial recess diameter of $2.54 \pm 0.51 \mathrm{~mm}(\mathrm{n}=74)$ was determined from a review of existing literature; subsequent measurements on CT data revealed a facial recess diameter of $2.54 \pm 0.5 \mathrm{~mm}(\mathrm{n}=23)$. The developed method was applied based on a previously described minimally invasive robotic system, whereby approximately $46.7 \%$ of the population could accommodate a safety region of three standard deviations above the mean drilling error, when utilizing a drill diameter of $1.8 \mathrm{~mm}$. Overall, the presented model allows the assessment of the applicability of a system on a wider population scale beyond examining only the ability to reach an arbitrary threshold accuracy 


\section{Acknowledgements}

218 This work was completed as part of the Nano-Tera initiative; the work was scientifically evaluated by 219 the Swiss National Science Foundation, financed by the Swiss Confederation and funded by Nano-

220 Tera.ch. The authors would like to acknowledge the Institute of Anatomy at the University of Bern for 221 providing the temporal bone specimens.

222 


\section{References}

224

1. Schipper J, Aschendorff A, Arapakis I, et al. Navigation as a quality management tool in cochlear implant surgery. J Laryngol Otol. 2004;118(10):764-770. doi:10.1258/0022215042450643.

2. Labadie RF, Mitchell J, Balachandran R, Fitzpatrick JM. Customized, rapid-production microstereotactic table for surgical targeting: description of concept and in vitro validation. Int $\mathrm{J}$ Comput Assist Radiol Surg. 2009;4(3):273-280. doi:10.1007/s11548-009-0292-3.

3. Eilers $\mathrm{H}$, Baron $\mathrm{S}$, Ortmaier $\mathrm{T}$, et al. Navigated, robot assisted drilling of a minimally invasive cochlear access. 2009 IEEE Int Conf Mechatronics. 2009;(April):1-6. doi:10.1109/ICMECH.2009.4957213.

4. Bell B, Gerber N, Williamson T, et al. In Vitro Accuracy Evaluation of Image-Guided Robot System for Direct Cochlear Access. Otol Neurotol. 2013;34:1284-1290. doi:10.1097/MAO.0b013e31829561b6.

5. Kratchman LB, Blachon GS, Withrow TJ, Balachandran R, Labadie RF, Webster RJ. Design of a bone-attached parallel robot for percutaneous cochlear implantation. IEEE Trans Biomed Eng. 2011;58(10):2904-2910. doi:10.1109/TBME.2011.2162512.

6. Kobler J-P, Schoppe M, Lexow GJ, et al. Temporal bone borehole accuracy for cochlear implantation influenced by drilling strategy: an in vitro study. Int J Comput Assist Radiol Surg. May 2014:1033-1043. doi:10.1007/s11548-014-0997-9.

7. Nau M, Pollmanns S, Schmitt R. Assessing the risk of minimally-invasive surgery: a metrological approach. In: Filtz J-R, Larquier B, Claudel P, Favreau J-O, eds. 16th International Congress of Metrology. Vol Les Ulis, France: EDP Sciences; 2013:07002. doi:10.1051/metrology/201307002.

8. McManus LJ, Dawes PJD, Stringer MD. Surgical anatomy of the chorda tympani: a micro-CT study. Surg Radiol Anat. February 2012. doi:10.1007/s00276-012-0941-z.

9. Bettman RHR, Appelman AMMF, van Olphen AF, Zonneveld FW, Huizing EH. Cochlear orientation and dimensions of the facial recess in cochlear implantation. ORL J Otorhinolaryngol Relat Spec. 2003;65(6):353-358. doi:10.1159/000076054. 
10. Rau TS, Kluge M, Prielozny L, Kobler J-P, Lenarz T, Majdani O. Auf dem Weg zur klinischen Anwendung: Eine Weiterentwicklung des automatisierten Insertionstools für CochleaImplantate. In: W. Freysinger, ed. 12. Jahrestagung Der Deutschen Gesselschaft Für ComputerUnd Roboter Assistierte Chirurgie. Vol Innsbruck; 2013:41-45.

11. Su WY, Marion MS, Hinojosa R, Matz GJ. Anatomical measurements of the cochlear aqueduct, round window membrane, round window niche, and facial recess. Laryngoscope. 1982;92(5):483-486. http://www.ncbi.nlm.nih.gov/pubmed/7078322.

12. Bielamowicz $\mathrm{S}$ a, Coker $\mathrm{NJ}$, Jenkins $\mathrm{H}$ a, Igarashi $\mathrm{M}$. Surgical dimensions of the facial recess in adults and children. Arch Otolaryngol Head Neck Surg. 1988;114(5):534-537. http://www.ncbi.nlm.nih.gov/pubmed/3355691.

13. Young YS, Nadol JB. Dimensions of the extended facial recess. Ann Otol Rhinol Laryngol. 1989;98(5 Pt 1):336-338. http://www.ncbi.nlm.nih.gov/pubmed/2719450.

14. Adad B, Rasgon BM, Ackerson L. Relationship of the facial nerve to the tympanic annulus: a direct anatomic examination. Laryngoscope. 1999;109(8):1189-1192. doi:10.1097/00005537199908000-00002.

15. Low WK. Surgical anatomy of the facial nerve in Chinese mastoids. ORL J Otorhinolaryngol Relat Spec. 61(6):341-344. doi:27696.

16. Hamamoto M, Murakami G, Kataura A. Topographical relationships among the facial nerve, chorda tympani nerve and round window with special reference to the approach route for cochlear implant surgery. Clin Anat. 2000;13(4):251-256. doi:10.1002/10982353(2000)13:4<251::AID-CA4>3.0.CO;2-E.

17. Gerber N, Bell B, Gavaghan K, Weisstanner C, Caversaccio MD, Weber S. Surgical planning tool for robotically assisted hearing aid implantation. Int $\mathrm{J}$ Comput Assist Radiol Surg. 2014;9(1):11-20. doi:10.1007/s11548-013-0908-5.

18. McRackan TR, Reda F a, Rivas A, et al. Comparison of cochlear implant relevant anatomy in $\begin{array}{lllll}\text { children } & \text { versus } & \text { adults. } & \text { Otol Neurotol. }\end{array}$ doi:10.1097/MAO.0b013e318245cc9f. 
19. Labadie RF, Balachandran R, Noble JH, et al. Minimally invasive image-guided cochlear implantation surgery: First report of clinical implementation. Laryngoscope. 2014;124(8):19151922. doi:10.1002/lary.24520.

20. Thom JJ, Carlson ML, Olson MD, et al. The prevalence and clinical course of facial nerve paresis following cochlear implant surgery. Laryngoscope. 2013;123(4):1000-1004. doi:10.1002/lary.23316.

21. Bhatia K, Gibbin KP, Nikolopoulos TP, O'Donoghue GM. Surgical complications and their management in a series of 300 consecutive pediatric cochlear implantations. Otol Neurotol. 2004;25(5):730-739. http://www.ncbi.nlm.nih.gov/pubmed/15354004.

22. Williamson TM, Bell BJ, Gerber N, et al. Estimation of tool pose based on force-density correlation during robotic drilling. IEEE Trans Biomed Eng. 2013;60(4):969-976. doi:10.1109/TBME.2012.2235439.

23. Bergmeier J, Daentzer D, Noll C, Majdani O, Ortmaier T, Kahrs LA. Towards endoscopic imageto-physical registration of mastoid cells and trabecula. In: 14. Jahrestagung Der Deutschen Gesellschaft Für Computerund Roboterassistierte Chirurgie. Vol ; 2015:43-48.

24. A. Sikandar, Gerber N, Williamson T, Caversaccio MD, Weber S, Gavaghan K. Intraoperative evaluation of an image guided robotically drilled trajectory for minimally invasive cochlear implantation. In: CARS 2015-Computer Assisted Radiology and Surgery - Proceedings of the 29th International Congress and Exhibition Barcelona, Spain, June 24-27. Vol ; 2015:101-102.

25. Ansó J, Dür C, Gavaghan K, et al. A Neuromonitoring Approach to Facial Nerve Preservation During Image-guided Robotic Cochlear Implantation. Otol Neurotol. 2016;37(1):89-98. doi:10.1097/MAO.0000000000000914.

26. Feldmann A, Anso J, Bell B, et al. Temperature Prediction Model for Bone Drilling Based on Density Distribution and In Vivo Experiments for Minimally Invasive Robotic Cochlear Implantation. Ann Biomed Eng. September 2015. doi:10.1007/s10439-015-1450-0. 


\section{Figure Captions}

304 Figure 1: Graphical representation of the concept. A safety region through the facial recess (constrained 305 by the facial nerve in yellow and chorda tympani in orange) can be defined by the diameter of the drill used $(\varnothing)$ and the accuracy characteristics of the system (mean $\mu$ and standard deviation $\sigma$ ). A confidence level for a specific facial recess diameter can be defined based on the number of standard deviations at which the border of the facial recess intersects with the safety region. In the above case, a safety region at a level of approximately $2 \sigma$ can be accommodated, resulting in a confidence value of approximately $98 \%$ when utilizing the defined system.

Figure 2: The facial recess diameter was defined as the sum of the drill diameter $\emptyset_{\text {Drill, }}$ the shortest distance between the drill and chorda tympani $D_{C h t}$ and the shortest distance between the drill and the facial nerve $D_{\mathrm{FN}}$. One can also consider the distance to the stapes ( $\mathrm{D}_{\mathrm{st}}$, the region inhabited by the stapes is represented as a cone), the incus and malleus (DIM) and the external auditory canal (DEAC).

Figure 3: Effect of changes in the portion of the treatable patient population as a result of different configurations in mean accuracy, standard deviation and drill diameter.

\section{Table Captions}

318 Table 1: Reported measurements of facial recess (FR) and extended facial recess (eFR).

319 Table 2: Relevant measurements of facial recess (FR) from literature, measurements completed within 320 this study and combination of all measurements.

321 Table 3: Treatable population for systems with different accuracy, precision and drill diameter values 322 and respecting one, two and three standard deviations above the mean targeting error. 
$\mu_{\text {acc }}$

$\mu_{\mathrm{acc}}+\sigma_{\mathrm{acc}}$ $\mu_{\mathrm{acc}}+2 \sigma_{\mathrm{acc}}$ $\mu_{\mathrm{acc}}+3 \sigma_{\mathrm{acc}}$ 


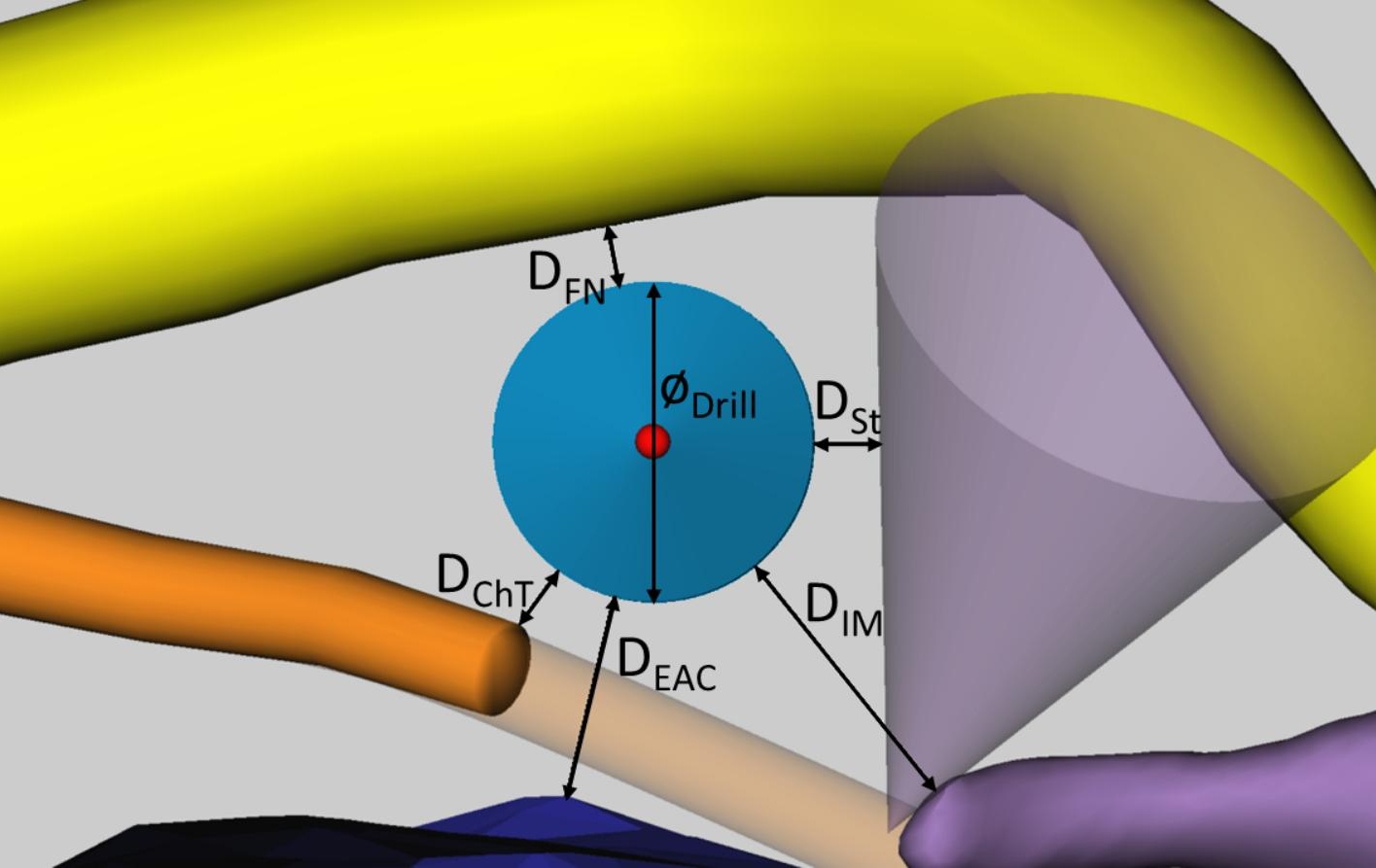




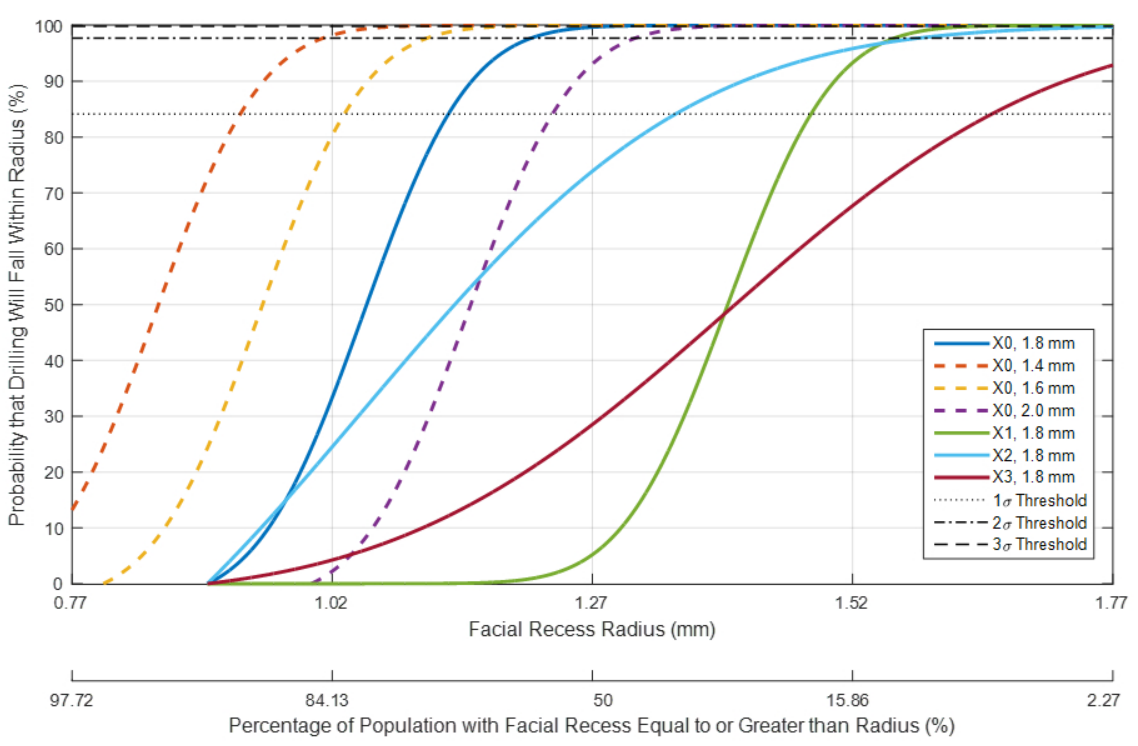


Table 1: Reported measurements of facial recess (FR) and extended facial recess (eFR).

\begin{tabular}{|c|c|c|c|c|c|}
\hline & Type & Measurement & Measurement Level & Diameter (mm) & $\begin{array}{l}\text { Number of } \\
\text { Samples }\end{array}$ \\
\hline Su (1982) & eFR & Histology & Pyramidal Eminence & $4.01 \pm 0.56$ & 356 \\
\hline $\begin{array}{l}\text { Bielamowicz } \\
\text { (1988) }\end{array}$ & $\begin{array}{l}\text { FR } \\
\text { eFR }\end{array}$ & Histology & $\begin{array}{l}\text { Oval Window } \\
\text { Round Window } \\
\text { Oval Window } \\
\text { Round Window }\end{array}$ & $\begin{array}{l}3.00 \pm 0.61 \\
2.65 \pm 0.63 \\
4.00 \pm 0.45 \\
3.42 \pm 0.46 \\
\end{array}$ & 20 \\
\hline Young (1989) & eFR & Histology & $\begin{array}{l}\text { Round window } \\
\text { Stapes }\end{array}$ & $\begin{array}{l}2.98 \pm 0.69 \\
3.91 \pm 0.78\end{array}$ & 97 \\
\hline Adad (1999) & eFR & Caliper & $\begin{array}{l}\text { Posterior Edge of } \\
\text { Tympanic Annulus }\end{array}$ & $3.78 \pm 0.87$ & 37 \\
\hline Low (1999) & eFR & Caliper & Pyramidal eminence & $4.4 \pm 0.94$ & 30 \\
\hline $\begin{array}{l}\text { Hamamoto } \\
(2000)\end{array}$ & FR & Pin Caliper & Round Window & $2.6 \pm 0.4$ & 22 \\
\hline $\begin{array}{l}\text { McManus } \\
(2012)\end{array}$ & FR & MicroCT & $\begin{array}{l}\text { Chorda tympani middle } \\
\text { ear entry }\end{array}$ & $2.9 \pm 0.7$ & 39 \\
\hline $\begin{array}{l}\text { Bettmann } \\
(2013)\end{array}$ & eFR & CT & $\begin{array}{l}\text { Round Window } \\
\text { Oval Window }\end{array}$ & $\begin{array}{l}4.5 \pm 1.3 \\
5.4 \pm 0.9\end{array}$ & 29 \\
\hline Rau (2013) & FR & СT & Basal Turn & $2.44 \pm 0.46$ & 32 \\
\hline
\end{tabular}


Table 2: Relevant measurements of facial recess (FR) from literature, measurements completed within this study and combination of all measurements.

\begin{tabular}{|l|l|l|l|l|}
\hline & Type & Measurement & Measurement Level & Diameter (mm) \\
\hline Bielamowicz (1988) & FR & Histology & Round Window & $2.65 \pm 0.63(\mathrm{n}=20)$ \\
\hline Hamamoto (2000) & FR & Pin Caliper & Round Window & $2.6 \pm 0.4(\mathrm{n}=22)$ \\
\hline Rau (2013) & FR & CT & Basal Turn & $2.44 \pm 0.46(\mathrm{n}=32)$ \\
\hline This work & FR & CT & Round Window & $2.54 \pm 0.5(\mathrm{n}=23)$ \\
\hline Combined & FR & - & - & $\mathbf{2 . 5 4 \pm 0 . 5 ( n = 9 7 )}$ \\
\hline
\end{tabular}


Table 3: Treatable population for systems with different accuracy, precision and drill diameter values and respecting one, two and three standard deviations above the mean targeting error.

\begin{tabular}{|c|c|c|c|c|c|c|}
\hline \multicolumn{4}{|c|}{ System characteristics $(\mathrm{mm})$} & \multicolumn{3}{|c|}{ Treatable Population at } \\
\hline System & $\mu_{a c c}$ & $\sigma_{a c c}$ & $\emptyset_{\text {Drill }}$ & $\mu_{a c c}+\sigma_{a c c}$ & $\mu_{a c c}+2 \times \sigma_{a c c}$ & $\mu_{a c c}+3 \times \sigma_{a c c}$ \\
\hline \multirow{4}{*}{$X_{0}$} & 0.15 & 0.08 & 1.4 & $91.3 \%$ & $85.1 \%$ & $76.4 \%$ \\
\hline & 0.15 & 0.08 & 1.6 & $83.1 \%$ & $73.9 \%$ & $62.5 \%$ \\
\hline & 0.15 & 0.08 & 1.8 & $71.2 \%$ & $59.5 \%$ & $46.8 \%$ \\
\hline & 0.15 & 0.08 & 2.0 & $56.3 \%$ & $43.6 \%$ & $31.6 \%$ \\
\hline$X_{1}$ & 0.5 & 0.08 & 1.8 & $20.1 \%$ & $12.3 \%$ & $6.9 \%$ \\
\hline$X_{2}$ & 0.15 & 0.25 & 1.8 & $43.2 \%$ & $12.9 \%$ & $1.7 \%$ \\
\hline$X_{3}$ & 0.5 & 0.25 & 1.8 & $6.4 \%$ & $0.59 \%$ & $0.02 \%$ \\
\hline
\end{tabular}

